\title{
Single centre experience on short bar technique for pectus excavatum
}

\author{
Hans Kristian Pilegaard ${ }^{1,2}$
}

${ }^{1}$ Department of Cardiothoracic and Vascular Surgery, Aarhus University Hospital, Aarhus, Denmark; ${ }^{2}$ Department of Clinical Medicine, Aarhus University, Aarhus, Denmark

Correspondence to: Hans K. Pilegaard. Department of Cardiothoracic and Vascular Surgery, Aarhus University Hospital, Skejby, Palle Juul-Jensens Boulevard 99, DK-8200 Aarhus N, Denmark. Email: pilegaard@dadlnet.dk.

\begin{abstract}
Background: Pectus excavatum (PE) is the most frequent anomaly of the anterior chest wall. Before 2001, all patients in Denmark were referred to the plastic and reconstructive surgeon for implantation of a subcutaneous silicone prosthesis, because the modified Ravitch procedure was not used. Since 2001, all patients have been treated with a modified Nuss procedure, which today has become the gold standard for correction of PE.

Methods: From September 2001 to March 2016, 1,713 patients have been operated by a modified Nuss procedure using the short bar at Aarhus University Hospital with a male-female ratio 6:1. The median age was 16 years (range 7-58 years). All operations were done in general anesthesia with epidural analgesia and all patients were operated by the same surgeon. All patients were seen routinely 6 weeks after surgery and the bars were removed after 3 years.

Results: Patients were younger than 18 years in 1,109 cases (65\%). The number of bars needed for optimal correction was one in 1,209 patients, two in 486 patients and three in 18 patients. The median length of bar changed from 11 inches to 10 inches during the study period. The annual number of procedures continued to rise during the study period [ 833 patients during the first 10 years and 880 patients in the last 6 years, though more patients received two bars in the later period, (34\%) versus (24\%)] and the proportion of patients older than 30 years increased from $7.7 \%$ to $10 \%$. The average duration of the operation was 36 minutes (range 12-270 minutes) and did not change significantly during the study period, change in operation time which was around half an hour. The median postoperative hospital stay decreased over time from 6 to 2 days. There was no mortality.

Conclusions: The modified Nuss procedure with the short bar technique is effective for the correction of PE. The results are stable with a low rate of bar malrotations, and may in most cases can be done in less than an hour in experienced hands.
\end{abstract}

Keywords: Pectus excavatum repair; Nuss; short bar; minimally invasive; surgery

Submitted Jul 06, 2016. Accepted for publication Sep 12, 2016.

doi: 10.21037/acs.2016.09.05

View this article at: http://dx.doi.org/10.21037/acs.2016.09.05

\section{Introduction}

The incidence of pectus excavatum (PE) is 1:3-400 in males and 4-6 times more frequent in males than in females (1). It is the most common anomaly of the anterior chest wall and more often seen in Marfan's syndrome. Approximately $40 \%$ of the patients have a family history.

Most patients seek treatment for cosmetic reasons but many patients also have cardiopulmonary complaints (2), which have shown to improve after surgical correction $(3,4)$.

Until Nuss published his first paper of the minimal invasive technique in 1998 (5) the Ravitch operation with modifications was the preferred operative treatment. In our hospital, however, this technique was never used. Instead, a silicone prosthesis was the treatment of choice until 2001, where the Nuss procedure changed the surgical treatment of the patients with $\mathrm{PE}$ from an open operation 


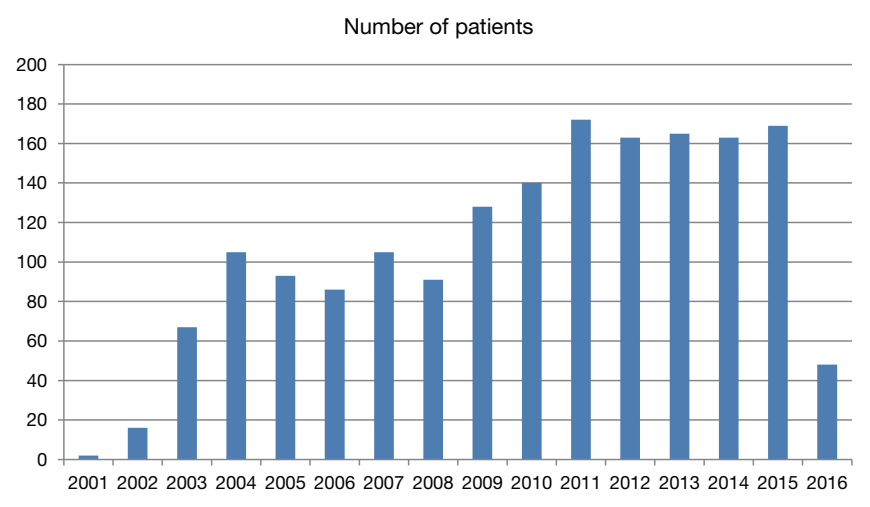

Figure 1 Number of pectus excavatum corrections each year at Aarhus University Hospital.

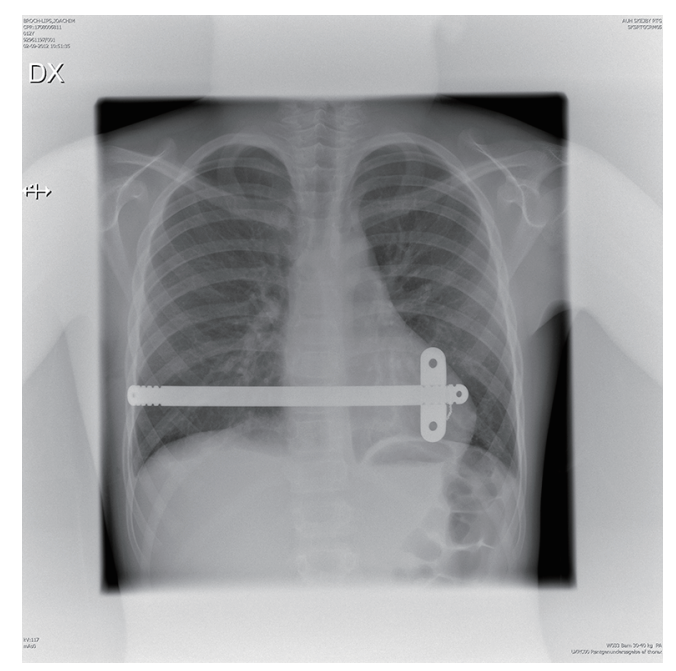

Figure 2 The standard asymmetric position of the bar.

with a sternotomy to a minimal invasive operation with two small lateral incisions on the chest. This minimal approach together with the fast diversification of the technique have considerably increased the number of patients in the last decade.

This paper describes our experience with the minimal invasive technique, since its introduction in our hospital in 2001.

\section{Methods}

In our department, the first patients were corrected with the Nuss procedure in September 2001 and the technique has since been used in all surgical procedures. The patients have been consecutively selected.

\section{Patients}

A total of 1,713 patients have been operated at Aarhus University Hospital, Skejby in the time period of September 2001 to March 2016. The annual numbers are shown in Figure 1. The median age at operation was 16 years (range 7-58 years), including 604 older than 18 years of age, and a male-female distribution of 1,457 to 256 . The age of the patients was younger than 20-25 years when we started, but with growing experience, even "very" old patients up to the age of 58 years have been treated in the same way with good results $(6,7)$. Today we have a minimum age of 11 years, and for girls it is advantageous to wait for the demarcation of the breast in order to place the incisions in the submammary fold. There have not been any specific inclusion and exclusion criteria. We increased the lower age limit from 7 to 11 years very early on in the study period, so only 18 patients below 11 years have been operated on. The depth of the pectus should be $2-3 \mathrm{~cm}$ measured from a slightly bent template depending of the age of the patient.

\section{Intervention}

Initially we used the technique described by Nuss (5) with the bar reaching from one midaxillary line to the other and with a stabilizer in each side. Very soon, we started to use a shorter bar to get the stabilizer closer to the hinge point $(8,9)$ in order to avoid flipping of the bar, and we reduced the numbers of stabilizers to one, which is most often positioned on the left side. During the first phase of using only one stabilizer, we used sutures to secure the bar to a rib on the opposite side of the stabilizer. In the last 6 years, however, we have not used additional sutures, and the bar has been placed asymmetrically on the chest (Figure 2). Using an asymmetric position enables the opposite side to cover two ribs (see art of operative technique in this journal). In most patients, the bars are inserted perpendicular to the patient, but in some patients an oblique bar or even crossed bars provide a better correction (Figures 3,4).

All patients have been seen in the outpatient clinic before surgery by the same surgeon, who performed all the procedures.

Most of the patients requested correction due to cosmetic reasons but approximately $60 \%$ also reported cardiopulmonary symptoms such as: breathlessness decreased and exercise tolerance. None of them had significant cardiac and/or respiratory comorbidities at baseline. They have been offered a correction if they had 


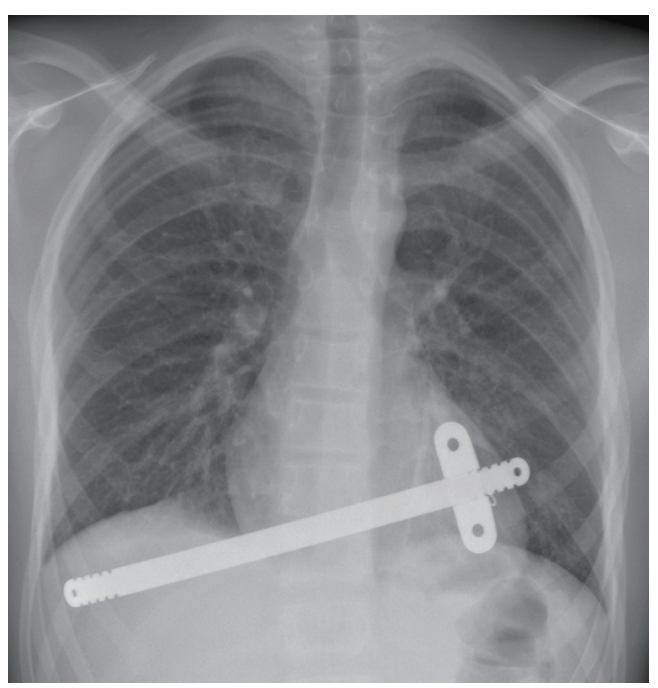

Figure 3 Oblique position of the bar.

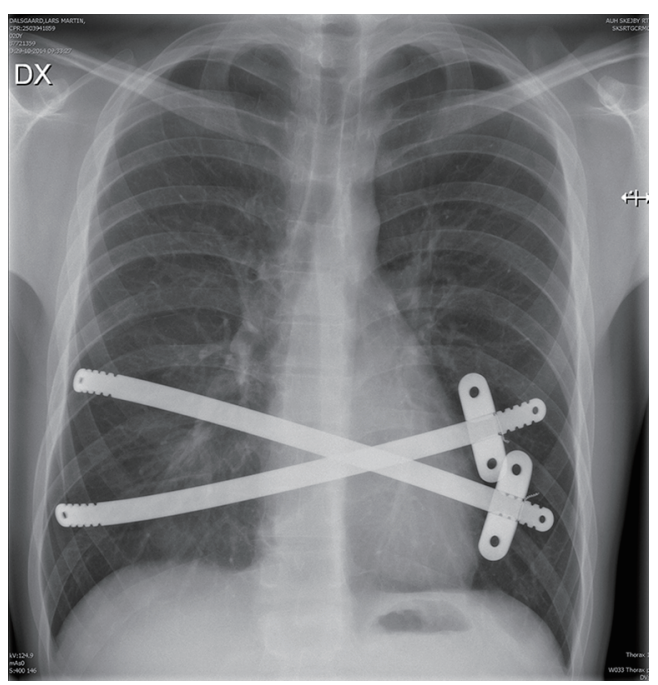

Figure 4 Crossed bars.

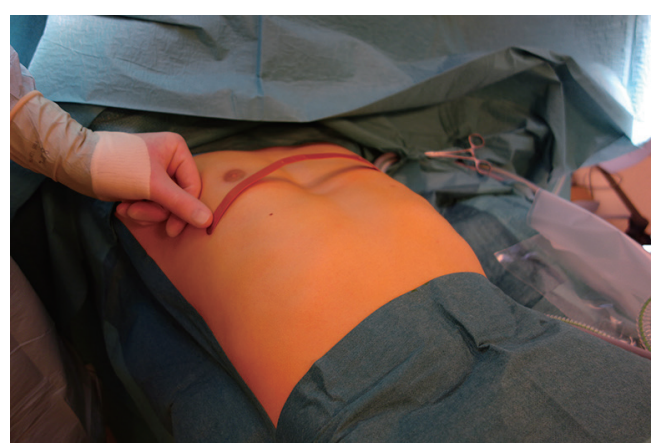

Figure 5 Showing how the template should be bent with some overcorrection. a PE with a depth of approximately $3 \mathrm{~cm}$ as measured by a curved template (Figure 5).

In the first years all patients had a CT-chest, but this was not used during the last 6-7 years. Echocardiography was only used if there was suspicion of cardiac pathology. None of our patients had a lung function testing. The Haller index has not been used routinely as an indicator for surgery.

Standard blood tests (hemoglobin, blood type and electrolytes) and $\mathrm{X}$-rays have been obtained before surgery and all patients have been instructed on a preoperative and postoperative exercise program.

The procedure is done under general anesthesia and we routinely place an epidural catheter preoperatively. Early on we used the epidural for 3-5 days; but we now remove it on the second postoperative day, Thereafter the patient receives oral analgesics for 4-5 weeks, including morphine for 10-14 days, ibuprofen for 3-4 weeks, and paracetamol for 4-5 weeks. Intravenous antibiotics are used for three days.

We obtain a chest X-ray routinely on postoperative day (10).

Restrictions in the first 6 weeks postoperatively are as follows: only carry $2 \mathrm{~kg}$ in front of the body, $5 \mathrm{~kg}$ on the shoulders, no sports, and no bicycle riding. The patients are advised to sleep on their back during this period.

As long as the bar(s) are in place, the patients are advised to avoid any heavy contact sports.

All patients are seen in the outpatient clinic 6 weeks postoperatively. Bar removal is performed 3 years later. If the patients have observed any problems in the meantime, they are advised to contact the hospital.

\section{Study design and data collection}

Data collection was performed retrospectively until 2010, after which data were collected prospectively.

Statistical data is presented as mean values, median, range and numbers.

\section{Results}

In total, we have used 2,235 bars in 1,713 patients, and the distribution related to age is shown in Table 1. Because there have been some changes in the surgical procedure over time, our results are divided into two time periods. The first time period, September 2001 to December 2010 and the second, January 2011 to March 2016, included to 833 and 880 patients, respectively. The mean length of bars was 


$\begin{aligned} & \text { Table } 1 \text { Showing the number of patients related to number of bars } \\
& \text { for the whole time period [2001-2016] }\end{aligned}$
\begin{tabular}{lllll} 
No. of bars & $<18$ years & $18 \leq$ years $\leq 30$ & $\geq 30$ years & Total \\
\hline $\begin{array}{l}\text { 1 bar } \\
2 \text { bars }\end{array}$ & 881 & 287 & 41 & 1,209 \\
3 bars & 0 & 157 & 101 & 486 \\
Total & 1,109 & 451 & 11 & 18 \\
\hline
\end{tabular}

Table 2 Showing the number of patients related to number of bars in the time period [2001-2010]. Percentage in []

\begin{tabular}{lllll}
\hline No. of bars & $<18$ years & $18 \leq$ years $\leq 30$ & $\geq 30$ years & Total \\
\hline 1 bar & $444[85]$ & $165[68]$ & $23[36]$ & $632[76]$ \\
\hline 2 bars & $81[15]$ & $74[30]$ & $39[61]$ & $194[23]$ \\
3 bars & 0 & $5[2]$ & $2[1]$ & $7[1]$ \\
\hline Total & 525 & 244 & 64 & 833 \\
\hline
\end{tabular}

Table 3 Showing the number of patients related to number of bars in the time period [2011-2016]. Percentage in []

\begin{tabular}{lllll}
\hline No. of bars & $<18$ years & $18 \leq$ years $\leq 30$ & $\geq 30$ years & Total \\
\hline 1 bar & $437[75]$ & $122[59]$ & $18[20]$ & $577[66]$ \\
\hline 2 bars & $147[25]$ & $83[40]$ & $62[70]$ & $292[33]$ \\
3 bars & 0 & $2[1]$ & $9[10]$ & $11[1]$ \\
\hline Total & 584 & 207 & 89 & 880 \\
\hline
\end{tabular}

11 inches (range, 7-15 inches) in the first time period, and 10 inches (range, 8-14 inches) in the second time period. Over time, there has been a tendency to use more bars in each patient (Tables 2,3), and the percentage of patients 30 years and older has increased. The mean duration of the correction was approximately 30 minutes, and was less than 60 minutes for over $90 \%$ of cases.

There has been no mortality.

The postoperative stay decreased over the study period. The first 100 patients stayed a median of 6 days in the hospital (range 4-29 days). Conversely, today, the median stay is 2 days and more than $85 \%$ of the patients are discharged in the afternoon of the second postoperative day. Nowadays, only $1.6 \%$ of patients stay longer than 4 days, compared with $46 \%$ during the early study period (Figure 6). In four patients, we combined the correction of $\mathrm{PE}$ with

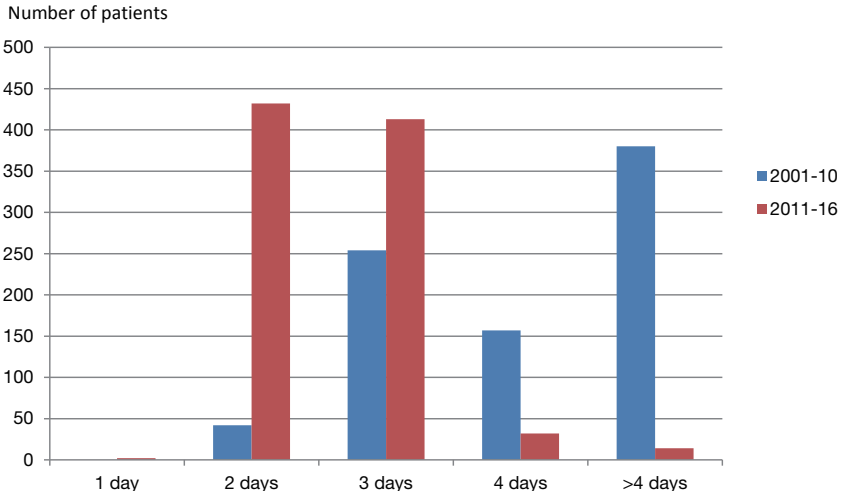

Figure 6 Showing the postoperative hospital stay for the two time periods.

a cardiac operation, David procedure $(\mathrm{n}=2)$ and composite graft $(\mathrm{n}=2)$. Complications are shown in Table 4.

Both of the sternotomies were done in re-do cases. One was caused by bleeding from the vena cava inferior and one was due to a tear in the right ventricle caused by an adhesion, from the primary Nuss correction.

The mean length of follow up was 36 months, at which point the patients were called for removal of the bars.

\section{Discussion}

The indications for surgery are related to cosmetics, quality of life and cardiopulmonary function. Cosmetic improvement and better quality of life are both rated highly by the patients and the parents (11), and better physical performance has been documented after correction $(3,4)$. More than $60 \%$ of the patients have cardiopulmonary symptoms preoperatively, as well as a $20 \%$ lower exercise capacity than their peers. The significant improvements in cardiac $(4,12)$ and pulmonary function $\left(\mathrm{FEV}_{1}\right)$ after surgery have been attributed to improved chest movements (13).

One of the challenges has been the stability of the bar. Initially, there were reports that approximately $15 \%$ of the bars flipped, but with application of a bar stabilizer, fixation of the stabilizers to the bars, and additional sutures, the rate of flipped bars was reduced to $1-2 \%$ (14). We have shown that a shorter bar with the stabilizer close to the hinge point has the same low frequency of rotation (Table 4). Only 11 bars out of 1,194 implanted $(<1 \%)$ flipped in the last time period.

The position of the trocar through the lateral chest wall at the level of the nipples facilitates the movement of 


\begin{tabular}{llll}
$\begin{array}{l}\text { Table } 4 \text { Number of complications in the time periods [2001-2010], } \\
\text { [2011-2016] and total study period }\end{array}$ & $\begin{array}{l}\text { 2001- } \\
2011-\end{array}$ & $\begin{array}{l}\text { Total } \\
2016\end{array}$ & \\
\hline Time period & 10 & 11 & 21 \\
\hline Bar rotation & 11 & 2 & 13 \\
\hline Dislocation & 15 & 1 & 16 \\
\hline Deep infection & 19 & 5 & 24 \\
\hline Removal of stabilizer because of pain & - & 1 & 1 \\
\hline Fractura sterni & 1 & 1 & 2 \\
\hline Sternotomy & 3 & 3 & 6 \\
\hline Removal of bar before time & 8 & 2 & 10 \\
\hline Pneumonia & 16 & 3 & 19 \\
\hline Pneumothorax & 6 & 0 & 6 \\
\hline Seroma & 4 & 1 & 5 \\
\hline Pleural effusion & 1 & 1 & 2 \\
\hline Bleeding demanding re-operation & 3 & 1 & 4 \\
\hline Bar end dropped into chest cavity & & & \\
\hline
\end{tabular}

the scope with no restrictions, creates a better view in the caudal part of the chest cavity without being restricted by the diaphragm, and minimizes the risk of injury to the diaphragm.

In most cases, it is not necessary to use a double-lumen tube for ventilation. If one expects problems, such as adhesions due to previous surgery or infection, the doublelumen tube is used to provide longer access time without ventilation problems. The number of bars has gradually increased as shown in Tables 2 and 3. In many patients, two bars may be implanted through the same incisions, and I prefer to have the stabilizers on the same side, which makes it easier for removal of the bars after 3 years. We have not had any complications with this technique, even if the stabilizers are placed on the same side, one above the other (Figure 4). In this case, the two stabilizers can be wired together to work as a bridge (15). Placing the stabilizer close to the hinge point also seems to fix the bar better. Seromas are complications thought to be caused by small movements of the bar irritating the tissue around the bar and stabilizer, but we have not seen any seromas in the second time period.

Pain treatment has always been considered a major problem regarding the Nuss-procedure. It is shown that a deeper PE or an older patient requires a higher dose of morphine (16), but with good preoperative information, an epidural catheter, and oral pain treatment 4-5 weeks postoperatively, this problem is prevented. The biggest problem for most patients is back pain when they sleep on their back during the first 6 weeks after the surgery.

Complications may be reduced when the department increases the number of procedures. The lower threshold of an acceptable case volume is controversial, but 2-3 cases a month is a reasonable number to maintain good experience for the surgeon and the staff. The rotation rate has been very low in the whole time period. Likewise, dislocations, which occurs when the bar moves laterally due to being positioned asymmetrically, have decreased. It is remarkable that the number of infections have also decreased, even though antibiotics have not changed for the last 10 years. We have never seen an acute infection after surgery. All infections were late, occurring after 4-10 weeks, and were treated by antibiotics. In addition, vacuum-assisted closure (VAC) was used in four patients. Two patients did not accept the prolonged treatment and requested removal of the barsystem before the set time.

\section{Conclusions}

The modified Nuss procedure using the short bar is effective in correcting PE. The results are very stable with a low bar rotation rate, and in most cases the surgery can be done in less than an hour in experienced hands.

\section{Acknowledgements}

None.

\section{Footnote}

Conflicts of Interest: Dr. Pilegaard is a consultant at Zimmer Biomet.

\section{References}

1. Cobben JM, Oostra RJ, van Dijk FS. Pectus excavatum and carinatum. Eur J Med Genet 2014;57:414-7.

2. Kelly RE Jr. Pectus excavatum: historical background, clinical picture, preoperative evaluation and criteria for operation. Semin Pediatr Surg 2008;17:181-93.

3. Kelly RE Jr, Cash TF, Shamberger RC, et al. Surgical repair of pectus excavatum markedly improves body image 
and perceived ability for physical activity: multicenter study. Pediatrics 2008;122:1218-22.

4. Maagaard M, Tang M, Ringgaard S, et al. Normalized cardiopulmonary exercise function in patients with pectus excavatum three years after operation. Ann Thorac Surg 2013;96:272-8.

5. Nuss D, Kelly RE Jr, Croitoru DP, et al. A 10-year review of a minimally invasive technique for the correction of pectus excavatum. J Pediatr Surg 1998;33:545-52.

6. Pilegaard HK, Licht PB. Routine use of minimally invasive surgery for pectus excavatum in adults. Ann Thorac Surg 2008;86:952-6.

7. Pilegaard HK. Extending the use of Nuss procedure in patients older than 30 years. Eur J Cardiothorac Surg 2011;40:334-7.

8. Pilegaard HK, Licht PB. Early results following the Nuss operation for pectus excavatum--a single-institution experience of 383 patients. Interact Cardiovasc Thorac Surg 2008;7:54-7.

9. Pilegaard HK, Licht PB. Can absorbable stabilizers be used routinely in the Nuss procedure? Eur J Cardiothorac Surg 2009;35:561-4.

10. Knudsen MR, Nyboe C, Hjortdal VE, et al. Routine postoperative chest $\mathrm{X}$-ray is unnecessary following the Nuss procedure for pectus excavatum. Interact Cardiovasc

Cite this article as: Pilegaard HK. Single centre experience on short bar technique for pectus excavatum. Ann Cardiothorac Surg 2016;5(5):450-455. doi: 10.21037/acs.2016.09.05
Thorac Surg 2013;16:830-3.

11. Jacobsen EB, Thastum M, Jeppesen JH, et al. Healthrelated quality of life in children and adolescents undergoing surgery for pectus excavatum. Eur J Pediatr Surg 2010;20:85-91.

12. Neviere R, Montaigne D, Benhamed L, et al. Cardiopulmonary response following surgical repair of pectus excavatum in adult patients. Eur J Cardiothorac Surg 2011;40:e77-82.

13. Redlinger RE Jr, Kelly RE, Nuss D, et al. Regional chest wall motion dysfunction in patients with pectus excavatum demonstrated via optoelectronic plethysmography. J Pediatr Surg 2011;46:1172-6.

14. Nuss D. Recent experiences with minimally invasive pectus excavatum repair "Nuss procedure". Jpn J Thorac Cardiovasc Surg 2005;53:338-44.

15. Park HJ, Kim KS, Moon YK, et al. The bridge technique for pectus bar fixation: a method to make the bar unrotatable. J Pediatr Surg 2015;50:1320-2.

16. Grosen K, Pfeiffer-Jensen M, Pilegaard HK. Postoperative consumption of opioid analgesics following correction of pectus excavatum is influenced by pectus severity: a singlecentre study of 236 patients undergoing minimally invasive correction of pectus excavatum. Eur J Cardiothorac Surg 2010;37:833-9. 